\title{
The effects of categorical and linguistic adaptation on binocular rivalry initial dominance
}

\author{
Vassilis Pelekanos $^{1}{ }^{*}$, Daphne Roumani ${ }^{1,2}$ and Konstantinos Moutoussis ${ }^{1}$ \\ ${ }^{1}$ Cognitive Science Laboratory, Department of Philosophy and History of Science, University of Athens, Athens, Greece \\ ${ }^{2}$ National and Kapodistrian University of Athens, Athens, Greece
}

\author{
Edited by: \\ Alexander Maier \\ Vanderbilt University, USA \\ Reviewed by: \\ Andreas Bartels, Max Planck \\ Institute for Biological Cybernetics, \\ Germany \\ Thomas Vergil Papathomas, \\ Columbia University, USA \\ *Correspondence: \\ Vassilis Pelekanos, Cognitive Science \\ Laboratory, Department of Philosophy \\ and History of Science, University of \\ Athens, University Campus, \\ Ano Ilissia, Athens 157 71, Greece. \\ e-mail:vpelekanos@gmail.com
}

\begin{abstract}
Binocular rivalry $(B R)$ is a phenomenon in which visual perception alternates between two different monocular stimuli. There has been a long debate regarding its nature, with a special emphasis on whether low- or high-level mechanisms are involved. Prior adaptation to one of the two monocular stimuli is known to affect initial dominance in the subsequent dichoptic presentation. In the present work, we have used three different types of adaptation in order to investigate how each one affects initial dominance during BR. In the first adaptation type, adapting to a stimulus identical to the one used during rivalry has led to its consequent suppression, verifying previous findings. The binocular presentation which we have used excludes the possibility of eye-adaptation, suggesting that it is the specific stimulus that the brain adapts to. In the second adaptation type, we find suppression effects following adaptation to stimuli belonging to the same category (face or house) but are different from the specific ones used in the following BR presentation. In the final adaptation type, in which the words "face" or "house" are used as adaptors, no statistically significant effect was found. These results suggest that perceptual selection can be directly influenced by the prior presentation of visual stimuli different to the ones used during BR, and thus support a higher-level, cognitive influence on the latter.
\end{abstract}

Keywords: binocular rivalry, high-level adaptation, embodied cognition, mental representations

\section{INTRODUCTION}

When two different visual stimuli are presented at corresponding retinal locations, one in each eye, perception alternates between them (or between parts of them - see below). This phenomenon is known as binocular rivalry (BR; e.g., Wheatstone, 1838; Blake, 1989). The unpredictable character of the perceptual alternations led Levelt (1965) to suggest that they are the result of a stochastic process following a gamma distribution, something that later became the "signature" of BR (Logothetis et al., 1996). In-between periods of complete dominance, perception has a piecemeal appearance (e.g., O'Shea et al., 1997) which, together with the dependence of perceptual alternations on low-level stimulus attributes (Breese, 1909) and the inability to control them by "the power of will" (Wheatstone, 1838, p. 386), have been taken by some as evidence toward an early, eye-competition mechanism, treating $\mathrm{BR}$ as the result of antagonism between opponent monocular neuronal populations (Levelt, 1965; Blake, 1989). Such a low-level account is also supported by studies showing that, during suppression, visual sensitivity is severely impaired in a way invariant to specific stimulus attributes, suggesting that suppression acts non-selectively upon the suppressed eye (e.g., Fox and Check, 1968; Fox and Rasche, 1969; Blake and Fox, 1974). Furthermore, when the two stimuli are interchanged between the eyes, observers perceive the previously suppressed stimulus (Blake et al., 1980). Such empirical findings support the notion of an early interocular competition, BR being the result of a continuous interplay of adaptation and recovery, mediated by reciprocal inhibition mechanisms (Blake, 1989). This notion, thus, predicts an early neuronal localization of the phenomenon, something partly confirmed by neuroimaging studies (e.g., Polonsky et al., 2000; Tong and Engel, 2001; Haynes et al., 2005).

Although piecemeal rivalry can be considered as indicating the existence of independent, local eye-zones of dominance, i.e., the footprint of a low-level mechanism (see Blake, 1989), it could also consist evidence for a higher-level control mechanism, combining inputs from the two eyes (Kovacs et al., 1996). Such an alternative, "stimulus-rivalry," theory treats BR as a high-level phenomenon, concerning central stimulus representations and thus permeable to top-down modulations (Walker, 1978; Logothetis, 1998). von Helmholtz was the first to suggest that rivalry is the result of attentional shifts and that, with practice, one can learn to prolong dominance of one of the stimuli (see Tong, 2001). Factors such as cultural background (Bagby, 1957), emotional content (Engel, 1956), and semantic meaning (Rommetveit et al., 1968) can modify the course of the effect, indicating an involvement of higherlevel processes on perceptual dominance. This idea is further supported by more recent and rigorous studies (e.g., Sobel and Blake, 2002; Andrews and Lotto, 2004; Mitchell et al., 2004; Bannerman et al., 2008; Costello et al., 2009). Treating BR as a high-level process deals with issues that the low-level, monocular competition, view cannot deal with: for example, issues like the co-existence of rivalry and stereopsis (Ogle and Wakefield, 1967; Julesz and Miller, 1975; Harrad et al., 1994), the integration of motion signals during rivalry (Carney et al., 1987; Carlson and He, 2000) and aftereffects that survive rivalry suppression (Lehmkuhle and Fox, 1975; but see Blake et al., 2006). Accordingly, single-unit recordings have 
shown that many neurons in the visual cortex still respond to the perceptually suppressed stimulus (Logothetis and Schall, 1989; Leopold and Logothetis, 1996; Sheinberg and Logothetis, 1997). There is also evidence for interocular grouping in BR percepts that combine similar stimulus parts from the two eyes (Kovacs et al., 1996). Perceptual alternations can, under specific conditions, be totally unaffected by eye-swapping of the stimuli (Logothetis et al., 1996). Additionally, and opposed to Wheatstone's (1838, p. 386) view, several studies show effects of directed attention and/or "will" on perceptual alternations (van Ee et al., 2005; Klink et al., 2008; de Graaf etal., 2011). This second view, therefore, regards BR as a competition between alternative stimulus representations, in which perceptual alternations engage higher-level cognitive mechanisms (Logothetis et al., 1996; Leopold and Logothetis, 1999).

In recent years, there has been an effort for a holistic explanation of BR, using an integrative approach between the two theories. The fact that there is evidence supporting both sides (see above) does not make it necessary that the two views should exclude each other. Co-existence and interaction between the two different suggested (high- and low-level) mechanisms is also a possibility. The stimulus rivalry account, although challenged as a phenomenon taking place under very specific, limited circumstances (Lee and Blake, 1999), has refreshed the idea of a high-level, cognitive approach (Blake, 2001), endorsing a posterior neuronal localization of rivalry (Logothetis, 1998). The basic ideas of eye-rivalry had to be refined and restated (see Lee and Blake, 1999; Blake, 2001). The need for an integrative approach became necessary, and the hypothesis of eye-competition had to change into an "eyeand-percept-competition hypothesis" (Papathomas et al., 1999). A possible reconciliation is to assume that we have to do with two distinct phenomena (Lee and Blake, 1999; Tong, 2001; Blake and Wilson, 2011), arising from distributed neural events occurring at multiple stages of visual processing (Blake and Logothetis, 2002; Ooi and He, 2003). Perhaps different mechanisms support different aspects of rivalry, such as the generation of transitions during rivalry and the maintenance of dominance and suppression (Tong et al., 2006). The current tendency for most researches is to account for both the eye/low-level and stimulus/high-level factors (Bhardwaj et al., 2008; van Boxtel et al., 2008; Alais et al., 2010; Bartels and Logothetis, 2010; Keliris et al., 2010).

Visual adaptation, on the other hand, is a process by which one can alter the response of the perceptual system to a stimulus (test), by first exposing it to another stimulus (adaptor; e.g., Lin and $\mathrm{He}, 2009)$. In addition to aftereffects due to low-level adaptation, such as adaptation to orientation (Blakemore and Campbell, 1969), or to direction of motion (Wohlgemuth, 1911), there is also higher-level adaptation to elements such as natural images, artworks, or even eye gaze and natural facial attributes like gender, ethnicity, and facial expressions (e.g., Clifford et al., 2007; Carbon and Ditye, 2011). The virtue of adaptation to isolate specific neuronal populations by decreasing responsiveness to subsequent stimulus presentation has made it a powerful tool for dissecting the neural processes of the visual hierarchy underlying BR (van Boxtel et al., 2008). Adaptation aftereffects have been used to study the non-conscious visual processing during BR (Lin and He, 2009), the possible neural locus of suppression (Lehmkuhle and Fox, 1975; Blake et al., 2006), the crucial factors underlying perceptual selection (Alais and Melcher, 2007; Hancock et al., 2008; van Boxtel et al., 2008), the cause of perceptual switches (Lankheet, 2006; Alais et al., 2010; Bartels and Logothetis, 2010), as well as the nature of the phenomenon per se (Blake, 1989; Wilson et al., 2001).

A common question regarding adaptation is whether it can influence BR's initial dominance. Also termed as "onset rivalry," initial dominance is a basic characteristic of the neural processes involved in perceptual selection, and can be biased by even a small shift in the balance between the processing of the two images (Brascamp et al., 2007). A straightforward way to manipulate initial dominance is "flash suppression" (Wolfe, 1984), during which, prior presentation of one stimulus results in the dominance of the other. More recent studies (e.g., Holmes et al., 2006; Hancock et al., 2008) have confirmed that when one or both eyes view an (adaptor) grating stimulus of a particular orientation before the presentation of that grating to one eye and an orthogonal (nonadapted) grating to the other eye, the non-adapted one gains initial perceptual dominance during BR. van Boxtel et al. (2008) have used complex (faces/houses) stimuli to confirm that adaptation leads to the suppression of the adapted stimulus and to the dominance of the non-adapted one. Additionally, they have shown that the effects of adaptation to gratings are limited to retinotopically matched locations, while, for the complex stimuli, such effects are evident in both retinotopically and spatiotopically matched locations. Furthermore, Hancock et al. (2008) have shown that in order for a stimulus to produce adaptation aftereffects, it must be consciously perceived, suggesting that the initial selection during BR involves later stages of visual processing. In the present study, we use adaptation of varying levels of complexity in order to test the top-down influence of higher-level mechanisms in BR.

\section{MATERIALS AND METHODS}

General note on participants:

In both experiments, native Greek speakers, undergraduate students at the University of Athens, participated for course credit. All reported normal or corrected-to-normal vision and were naïve about the purpose of the study. All experiments were carried out in accordance to the national regulations and legislations of the University of Athens, and informed consent was obtained from all the participants.

\section{EXPERIMENT 1 \\ Participants}

This experiment used 25 participants (17 females, mean age $=24.7, \mathrm{SD}=6.8$ ).

\section{Material}

For the binocular presentation of stimuli, we have used a ScreenScope mirror stereoscope SA200, mounted in front of the stimulus-presentation monitor. Stimuli consisted of gray $\left(33 \mathrm{~cd} / \mathrm{m}^{2}\right)$ sketches of face and house images (two of each) as well as the words "Face" and "House" written in the Greek language. All stimuli were presented against a black $\left(1 \mathrm{~cd} / \mathrm{m}^{2}\right)$ background. All the BR-testing periods across conditions consisted of a particular face and a particular house image presented dichoptically, and thus competing for perceptual dominance. The rest of our 
stimuli were used as adaptors, depending on the condition (see Design and Procedure). In order to enable fusion and alignment, so that the two monocular stimuli would fall on corresponding retinal locations, a red $\left(30 \mathrm{~cd} / \mathrm{m}^{2}\right)$ square aperture $\left(2.86^{\circ}\right.$ of visual angle) surrounding the stimuli and a fixation cross were also used. In order to secure fixation and eye-alignment in each trial, adaptation and BR periods were intermediated by the presentation of the aperture and the fixation cross alone. Stimuli were generated using Adobe Illustrator and presented on a 17 -inch LCD HP monitor $(1280 \times 1024$ pixels at $60 \mathrm{~Hz})$ using DMDX software (Forster and Forster, 2003). Luminance was measured using a Gossen Mavolux 5032C photometer. Experiments were conducted in a dark room, and participants viewed the computer monitor through the stereoscope, resting on a custom-made chin holder at a distance of $40 \mathrm{~cm}$.

\section{Design and Procedure}

Figure 1 shows a schematic example of the procedure. Across conditions, the BR-testing period was preceded by an adaptation period, in which a single stimulus (adaptor) was presented to both eyes. Adaptation duration was $10 \mathrm{~s}$, except for the "Linguistic" (see below) conditions, in which it was $1 \mathrm{~s}$. After the adaptation period, the adaptor stimulus was removed leaving only the aperture and fixation cross for $50 \mathrm{~ms}$ on the screen, accompanied by a beep (at $480 \mathrm{~Hz}$ ) used to indicate that the testing period was about to begin. This was, in turn, followed by $5 \mathrm{~s}$ of dichoptic presentation of a face and a house, during which, participants had to give their response. Their task was to keep fixation and indicate their perceptual state (face or house) using continuous button presses. They were instructed to respond even when the suppression of the less-visible stimulus was not complete. Although we have included only initial dominance in our analysis, participants had to keep pressing the appropriate buttons during the whole 5-s period of dichoptic presentation.

We have used seven different experimental conditions, belonging to four different main types of adaptation sessions (control, same, categorical, and linguistic). In the Control condition, designed to provide baseline performance, BR was preceded

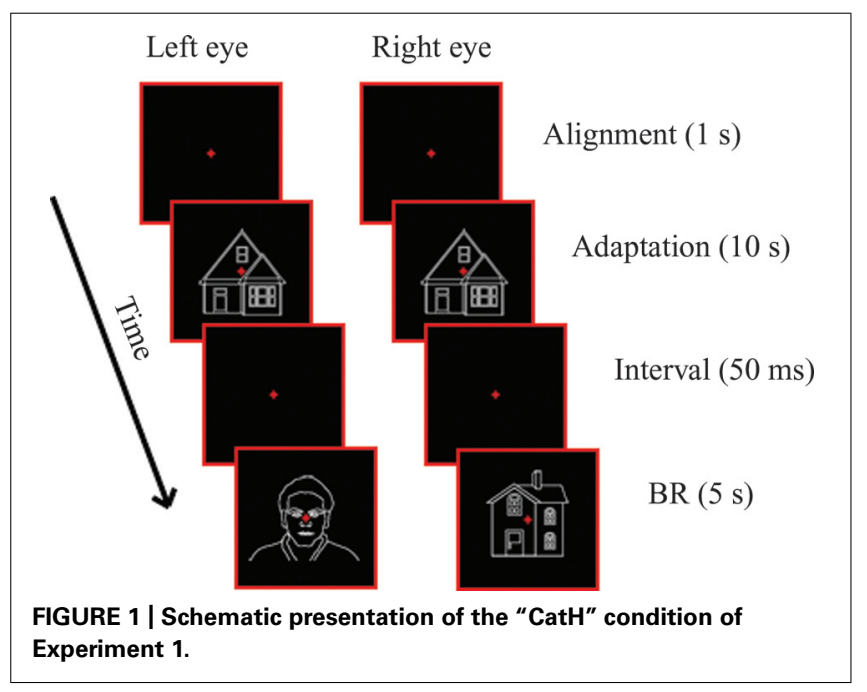

by adaptation to the aperture and fixation cross alone. In the Same Face Adaptation ("SameF") and Same House Adaptation ("SameH") conditions, BR was preceded by adaptation to the standard face or house images (used also during BR) respectively. In the Categorical Face Adaptation ("CatF") and Categorical House Adaptation ("CatH") conditions, BR was preceded by adaptation to a face or a house image, different to the ones used during BR testing. This design let us examine whether high-level adaptation, i.e., adaptation to different tokens of the categories faces/houses, could result in the suppression of the adapted stimulus category and the dominance of the non-adapted one. Finally, in the Linguistic Face Adaptation ("LingF") condition, BR was preceded by the word "Face" and in the Linguistic House Adaptation ("LingH") by the word "House." The purpose of these two conditions was to examine whether the presentation of a single word referring to a stimulus category would be able to induce suppression effects similar to the ones produced by adaptation to images.

In order to keep a steady adaptation level, conditions were completed in separate blocks consisting of 30 trials each, apart from the "LingF" and "LingH" conditions which were presented within the same block in random order (60 trials: 30 with "Face" adaptor and 30 with "House" adaptor). To avoid any eye-of-origin effects, a counterbalanced design was used during all BR periods: in half the trials of each condition, the face image was presented to the left eye and the house image to the right, while in the other half, they were presented reciprocally. The blocks were attended in random order and participants made a break of up to $10 \mathrm{~min}$ between blocks. Before the experiment started, each participant attended a 20 -trial practice block (with no adaptation) in half of which, the face was presented alone, and in the other half the house alone, always binocularly. The purpose of this was to familiarize participants with the appropriate button presses for each percept. During practice, the program provided feedback presenting the message "CORRECT," written in green, for correct answers and "WRONG," written in red, for wrong answers.

\section{EXPERIMENT 2}

\section{Participants}

This experiment used another 10 native Greek speakers (nine females, mean age $=27.2, \mathrm{SD}=7.3$ ).

\section{Design and procedure}

A possible objection to the design of Experiment 1 would be that, in the categorical conditions, participants adapt to some low-level characteristics of the stimuli, which are different between faces and houses. In particular, although the two categories were equalized in terms of their average size, luminance, and contrast, one could argue that the houses contained more straight than curved lines, with the opposite being true for the faces. For this reason, we have conducted the present, control experiment using, as adaptors, scrambled versions of our stimuli, which were no longer recognizable as proper faces and houses. If the effects that we have observed in Experiment 1 (see Results) were due to low-level factors, such a manipulation should make no difference. On the other hand, if adaptation was of a higher-level categorical type, no significant effects should be observed with the scrambled adaptors. Additionally, in Experiment 1, the linguistic conditions were, contrary to 
the rest of the conditions, randomized within the same block, and the adaptation duration was only $1 \mathrm{~s}$. In order to make sure that it was not because of these methodological differences that the linguistic conditions gave no significant effects (see Results), in Experiment 2, we have separated the two linguistic conditions into different blocks and have increased the time-course of adaptation to $10 \mathrm{~s}$. Thus, the linguistic conditions here are similar and directly comparable to the categorical ones. In particular, this experiment consisted of the following conditions: "Control," "CatF," "CatH," "LingF" and "LingH." Materials, testing stimuli, and the general procedure were identical to Experiment 1, apart from the following two differences: (1) the adaptors in "CatF" and "CatH" were turned upside down and their parts randomly scrambled, to the point that they did no more resemble face and house caricatures. (2) The two linguistic conditions were presented in separate blocks, with an adaptation duration of $10 \mathrm{~s}$.

\section{RESULTS \\ EXPERIMENT 1}

Table 1 and Figure 2 show the percentage of trials in which the initial percept was face, as a function of the condition, averaged across all 25 participants. In the control condition, used to reveal any biases in favor of the one percept or the other, the face stimulus dominated first in $52.7 \%$ of the trials ( $p=0.01$, binomial distribution). This suggests that, without any stimulus-adaptation, there was a slight but statistically significant preference for faces, which was used as a baseline in order to evaluate perceptual biases after adaptation to different types of stimuli.

A 3 (adaptation type: same/categorical/linguistic) $\times 2$ (adaptor stimulus: face/house) repeated-measures ANOVA for the initial face dominance showed a non-significant main effect of adaptation type [same: $M=52.2 \%, \mathrm{SE}=1.802$; categorical: $M=51.6 \%$, $\mathrm{SE}=2.049$; linguistic: $M=56 \%, \mathrm{SE}=2.339 ; F(2,48)=2.784$, $\mathrm{MSE}=102.259, p=0.072]$. The lack of adaptation type main effect suggests that, on average, participants adopted similar response strategies, no matter whether they were adapted to same, categorical, or linguistic face/house stimuli. On the other hand, there was a significant main effect of adaptor stimulus on face initial dominance [face adaptors: $M=47.5 \%, \mathrm{SE}=2.278$; house adaptors: $M=59.1 \%, \mathrm{SE}=2.151 ; F(1,24)=17.101$,

Table 1 | Summary statistics for face initial dominance across conditions, for Experiment 1.

\begin{tabular}{llll}
\hline Condition & $\boldsymbol{N}$ & $\boldsymbol{M}$ (SD) & SE \\
\hline Control & 25 & $52.7(8.9)$ & 1.8 \\
SameF & 25 & $38.9(15.8)$ & 3.2 \\
SameH & 25 & $65.5(13.7)$ & 2.7 \\
CatF & 25 & $46.3(12.2)$ & 2.4 \\
CatH & 25 & $56.9(13.1)$ & 2.6 \\
LingF & 25 & $57.2(15.3)$ & 3.1 \\
LingH & 25 & $54.8(15.9)$ & 3.2 \\
\hline
\end{tabular}

$S E= \pm 1$ standard error of the mean, across participants.

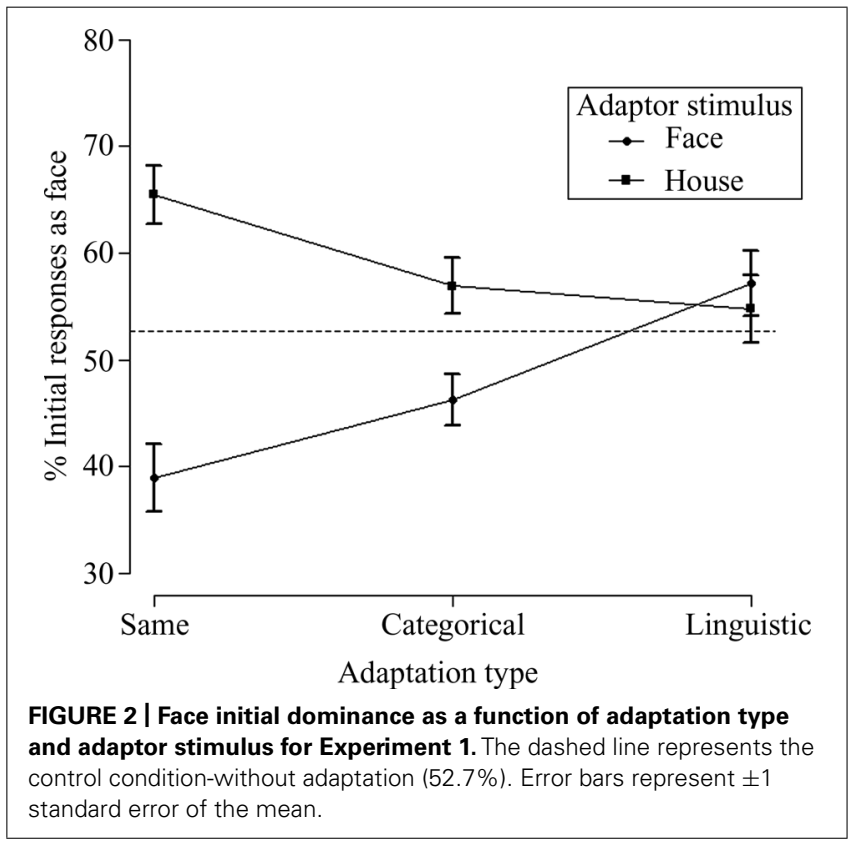

MSE $=295.074, p<0.001]$ showing that adaptation to a face stimulus resulted in a decrease of face initial dominance and in an increase of house initial dominance during BR, whereas the opposite was the case after adaptation to a house stimulus. Also, there was a significant interaction between adaptation type and adaptor stimulus, $F(2,48)=17.212$, MSE $=152.463, p<0.001$, showing that not all types of adaptation were equally effective.

In order to further investigate the interaction between the type of adaptation and adaptor stimulus, we conducted simple effects analyses: paired comparison analysis within adaptation type categories, showed that in significantly more BR trials face gained initial dominance after adapting to "SameH" $(M=65.5 \%$, $\mathrm{SE}=2.747)$ compared to "SameF" $(M=38.9 \%, \mathrm{SE}=3.160)$, $t(24)=5.645, p=0.003$. More interestingly, in significantly more BR trials face gained initial dominance after adapting to "CatH" $(M=56.9 \%, \mathrm{SE}=2.617)$ compared to "CatF" $(M=46.3 \%$, $\mathrm{SE}=2.436), t(24)=3.60, p=0.003$. There was not a significant difference for the face initial dominance between "LingH" $(M=54.8 \%, \mathrm{SE}=3.180)$ and "LingF" $(M=57.2 \%, \mathrm{SE}=3.065)$, $t(24)=0.580, p=0.567$. (All $p$-values were adjusted for multiple comparisons). As shown in Figure 2, the strongest effect of adaptation on initial dominance was observed in the same adaptation type, followed by the categorical adaptation type, with no statistically significant differences observed in the linguistic type. In both the same and categorical, adapting to a face resulted in the suppression of the face in favor of the rivaling house stimulus during $\mathrm{BR}$, whereas adapting to the corresponding house stimulus had the opposite effect. One-way repeated-measures ANOVA with different adaptation types to face stimuli as factors revealed a significant main effect of face-adaptation type: because the sphericity assumption was violated, we used Greenhouse-Geisser correction $(\epsilon=0.76): F(1.522,36.525)=17.335$, MSE $=160.142$, $p<0.001$. Pair-wise comparisons revealed a significant difference between "SameF" and "CatF" (face dominance was less in "SameF," 
$p=0.007$ ), a significant difference between "SameF" and "LingF" (face dominance was less in "SameF," $p<0.001$ ), and a significant difference between "CatF" and "LingF" (face dominance was less in "CatF," $p=0.008$ ). Conversely, one-way repeated-measures ANOVA with different adaptation types to house stimuli as factors, also revealed a significant main effect of house-adaptation type: $F(2,48)=5.994$, MSE $=132.864, p=0.005$. Pair-wise comparisons revealed a significant difference between "SameH" and "CatH" (face dominance was greater in "SameH," $p=0.039$ ) and a significant difference between "SameH" and "LingH" (face dominance was greater in "SameH," $p=0.007)$. There was no significant difference between "CatH" and "LingH" $(p=1)$.

To evaluate the effect that each adaptation condition had, we investigated whether there were any differences in initial dominance between the control and the other conditions. Paired comparisons showed a statistically significant difference between Control and "SameF" ( $p<0.0001)$, between Control and "SameH" $(p<0.0001)$, and between Control and "CatF" ( $p=0.037)$. These results show that these three adaptation conditions significantly changed the balance of initial dominance in perception, compared to what it was without adaptation.

\section{EXPERIMENT 2}

No effect was found in either paired comparison analysis of the conditions ["CatF" vs "CatH" comparison: $t(9)=1.208, p=0.258$; “LingF" vs “LingH" comparison: $t(9)=0, p=1.0$; see also Table 2].

\section{DISCUSSION}

In the present study we have shown that adaptation to a visual stimulus results in its initial suppression in a subsequent dichoptic presentation. In the "same" adaptation type, the stimuli used during adaptation and BR were identical, confirming what Wolfe (1984) has previously termed as "flash suppression." A difference between the present result and that of Wolfe's is that we (as well as Holmes et al., 2006; Hancock et al., 2008) have presented the adaptor stimulus binocularly. In this way, the observed suppression cannot be accounted for by eye-specific adaptation, but rather reflects the adaptation of the system to the specific stimulus, indirectly supporting a higher-level view of the mechanisms underlying perceptual selection during dichoptic presentation (Walker, 1978; Logothetis, 1998). However, despite the exclusion of an eye-adaptation effect, the possibility that the brain adapts

Table 2 | Summary statistics for face initial dominance across conditions, for Experiment 2.

\begin{tabular}{llll}
\hline Condition & $\boldsymbol{N}$ & $\boldsymbol{M}$ (SD) & SE \\
\hline Control & 10 & $49.7(5.5)$ & 1.8 \\
CatF & 10 & $42.0(12.2)$ & 3.9 \\
CatH & 10 & $47.7(11.2)$ & 3.5 \\
LingF & 10 & $47.3(11.2)$ & 3.5 \\
LingH & 10 & $47.3(11.6)$ & 3.7 \\
\hline
\end{tabular}

$S E= \pm 1$ standard error of the mean, across participants. to low-level characteristics of the specific stimulus still remains. Interestingly, our data also showed perceptual suppression when the adaptor stimulus belonged to the same category as the test stimulus, but was not identical to it: adaptation to faces and houses different to the ones subsequently presented dichoptically, were found to bias perception in favor of the other category. By using scrambled versions of the adaptor stimuli, and thus resembling all the low-level characteristics, we have verified that the observed adaptation effects were indeed categorical, i.e., of a higher, more abstract level. The fact that the presentation of one type of images can affect the fate of other images of the same type is in agreement with higher-level, cognitive, mechanisms being responsible for perceptual selection, when a controversy between the information from the two eyes has to be dealt with.

Along this line of thought, we have assumed the possibility that adaptation to an even higher, semantic level could perhaps influence perceptual selection during BR as well. Embodied theories for language comprehension regard words as the cues enabling the neuronal reactivation of the perceptual experience of the words' referents (e.g., Glenberg, 1997; Barsalou, 1999; Fincher-Kiefer, 2001; Glenberg and Kaschak, 2002; Zwaan, 2004; Gallese and Lakoff, 2005). The idea that linguistic representations reside in perceptual representations is supported by several behavioral studies (e.g., Stanfield and Zwaan, 2001; Zwaan et al., 2002, 2004; Yaxley and Zwaan, 2007), whereas a direct influence of language on perceptual sensitivity has also been shown by Meteyard et al. (2007), and Pelekanos and Moutoussis (in press). Moreover, neuroimaging evidence suggests that linguistic stimuli (words or sentences) activate neuronal circuits which are also selectively active during actions or perceptions involving the linguistic stimuli's referents (e.g., Isenberg et al., 1999; Pulvermüller, 1999, 2005; Speer et al., 2009). Similar, and especially interesting brain-imaging results have also been obtained by a recent fMRI study, using linguistic material specifically related to faces and places: Aziz-Zadeh et al. (2008) showed that listening to sentences which are related to faces can modulate neuronal activity in the left hemisphere's fusiform face area (FFA), while listening to sentences related to places modulates neuronal activity in the left hemisphere's parahippocampal place area (PPA). FFA has been found to respond more strongly to the perception of faces compared to a great variety of other, non-face stimuli, and PPA has been found to respond strongly during the perception of place and house stimuli, but not of face stimuli (see below). Based on the studies reviewed above, in the linguistic conditions of the present study, we briefly presented the words "face" or "house" before the dichoptic presentation of face and house image stimuli, with the assumption that an imagerylike, perceptual representation of the linguistic face/house adaptor could be sufficient to evoke suppressive effects. On the other hand, facilitation following prior presentation of images has been also reported previously, especially when the adaptor/prime is presented briefly (e.g., Brascamp etal., 2007) as was the case with our linguistic adaptors in Experiment 1. Furthermore, the semantic meaning of words presented prior to dichoptic stimulation has been also shown to have facilitative-priming effects: the word "hell" was more likely to gain perceptual dominance during BR between that, and a typographically similar word like "tell," when the BR period was preceded by the binocular presentation 
of a context-related word like "devil" (Rommetveit et al., 1968). Similarly, semantically related words have been found to come out of suppression faster than unrelated words, when a primeprecedent word is presented (Costello et al., 2009). Taking everything into consideration, it would seem quite reasonable to assume that prior presentation of a word would affect, by any means, perceptual selection mechanisms during BR. However, no statistical significance effect of language on perceptual selection was found in our study.

Our main, novel finding remains that of the effect of a general type, categorical adaptation on the perceptual result of a subsequent dichoptic presentation. In the domain of BR, a somehow similar result has been reported by Wolfe (1984): by changing the spatial frequency and luminance of the gratings used, he showed that suppression is not dependent on a perfect match between the adaptor and the test stimuli. The fact that he used low-level stimuli, however, does not indicate any higher-level, categorical influence on BR-a general adaptation of early orientation-selective channels could easily account for his result. In fact, since monocular adaptation was used, Wolfe's result could also be accounted for by eye-specific adaptation. In the present experiments, we show that a general, categorical adaptation to complex, high-level stimuli such as faces and houses can affect the outcome of perceptual competition during BR. A similar adaptation effect on ambiguous images has been reported recently: when participants were first adapted to a face or a hand and then presented with a combination of both, they perceived the non-adapted stimulus (Cziraki et al., 2010). Interestingly, the ambiguous image used consisted of a face and a hand different from the adaptors, showing a more abstract, categorical adaptation aftereffect, as is the case in the present study. The fact that such a categorical adaptation had an influence in our experiments as well supports the notion of a higher-level nature of $\mathrm{BR}$, in which top-down cognitive interactions play a role in perceptual selection (Walker, 1978; Logothetis, 1998). Furthermore, this influence is not only based on "simple," perceptual representations, but on cognitive, knowledge-based representations as well.

\section{REFERENCES}

Aguirre, G. K., Zarahn, E., and D’Esposito, M. (1998). An area within human ventral cortex sensitive to "building" stimuli: evidence and implications. Neuron 21, 373-383.

Alais, D., Cass, J., O'Shea, R. P., and Blake, R. (2010). Visual sensitivity underlying changes in visual consciousness. Curr. Biol. 20, 1362-1367.

Alais, D., and Melcher, D. (2007). Strength and coherence of binocular rivalry depends on shared stimulus complexity. Vis. Res. 47, 269-279.

Andrews, T. J., and Lotto, R. B. (2004). Fusion and rivalry are dependent on the perceptual meaning of visual stimuli. Curr. Biol. 14, 418-423.

Anstis, S. M., Verstraten, F. A. J., and Mather, G. (1998). The motion aftereffect. Trends Cogn. Sci. 2, 111-117.

A well-known aftereffect caused by prolonged adaptation is the motion aftereffect (MAE): after adaptation to a motion of a particular direction, the subsequent viewing of a stationary scene will be perceived as moving to the opposite direction (e.g., Anstis et al., 1998). MAE was originally explained by Sutherland (1961) who suggested that the direction in which something is perceived to move may depend on the ratios of firing of cells sensitive to opposite directions of motion: after prolonged adaptation to a specific direction, a stationary scene "would produce less firing in the cells which had just been stimulated than normally, hence movement in the opposite direction would be seen to occur" (Sutherland, 1961, p. 227) ${ }^{1}$. This explanation was inspired by Hubel and Wiesel's (1959) observation of opponent populations of directionally selective neurons in cat's cortex. Would a similar account be possible for the aftereffects observed in our study? The complex stimuli that we and others (e.g., van Boxtel et al., 2008) have used are known to activate specific, "higher" brain regions: the human FFA, in the fusiform gyrus, which responds more strongly to the perception of faces compared to a great variety of other, non-face stimuli (e.g., Puce et al., 1996; Kanwisher et al., 1997, 1999; McCarthy et al., 1997), and the PPA, which responds strongly during the perception of place and house stimuli, but not of face stimuli (Aguirre et al., 1998; Epstein and Kanwisher, 1998). Furthermore, such specificity is also present during BR (Tong et al., 1998). Adaptation of these areas, following the presentation of various face and house adaptor stimuli, could perhaps, theoretically explain the subsequent result on perceptual selection. However, such a hypothesis, suggesting an opponent mechanism between FFA and PPA, remains highly speculative, since there is no known anatomical connection or activity "comparison" between the two areas.

\section{ACKNOWLEDGMENTS}

We are grateful to Ioanna Roumani for the generation of the stimuli, and to Emmanouil Konstantinidis for his help with the statistical analysis. ${ }^{1}$ We recognize that the explanation for MAE may require a lot more complex models
(e.g., Stocker and Simoncelli, 2009) than the one suggested by Sutherland (1961).

Bhardwaj, R., O'Shea, R., Alais, D., and Parker, A. (2008). Probing visual consciousness: rivalry between eyes and images. J. Vis. 8, 1-13.

Blake, R. (1989). A neural theory of binocular rivalry. Psychol. Rev. 96, $145-167$.

Blake, R. (2001). A primer on binocular rivalry, including current controversies. Brain Mind 2, 5-38.

Blake, R., and Fox, R. (1974). Binocular rivalry suppression: insensitive to spatial frequency and orientation change. Vis. Res. 14, 687-692.

Blake, R., and Logothetis, N. K. (2002). Visual competition. Nat. Rev. Neurosci. 3, 13-21.

Blake, R., Tadin, D., Sobel, K., Raissian, T., and Chong, S. C. (2006). Strength of visual adaptation depends on visual awareness. Proc. Natl. Acad. Sci. U.S.A. 103, 4783-4788.
Blake, R., Westendorf, D. J., and Overton, R. (1980). What is suppressed during binocular rivalry? Perception 9, 223-231.

Blake, R., and Wilson, H. (2011). Binocular vision. Vis. Res. 51, 754-770.

Blakemore, C., and Campbell, F. W. (1969). Adaptation to spatial stimuli. J. Physiol. 200, 11-13.

Brascamp, J. W., Knapen, T. H. J., Kanai, R., van Ee, R., and van den Berg, A. V. (2007). Flash suppression and flash facilitation in binocular rivalry. $J$. Vis. 7, 1-12.

Breese, B. B. (1909). Binocular rivalry. Psychol. Rev. 16, 410-415.

Carbon, C. C., and Ditye, T. (2011). Sustained effects of adaptation on the perception of familiar faces. J. Exp. Psychol. Hum. Percept. Perform. 37, 615-625. 
Carlson, T. A., and He, S. (2000). Visible binocular beats from invisible monocular stimuli during binocular rivalry. Curr. Biol. 10, 1055-1058.

Carney, T., Shadlen, M., and Switkes, E. (1987). Parallel processing of motion and colour information. Nature 328 , 647-649.

Clifford, C. W., Webster, M. A., Stanley, G. B., Stocker, A. A., Kohn A., Sharpee, T. O., and Schwartz, O. (2007). Visual adaptation: neural, psychological and computational aspects. Vis. Res. 47, 3125-3131.

Costello, P., Jiang, Y., Baartman, B., McGlennen, K., and He, S. (2009). Semantic and subword priming during binocular suppression. Conscious. Cogn. 18, 375-382.

Cziraki, C., Greenlee, M. W., and Kovacs, G. (2010). Neural correlates of high-level adaptation-related aftereffects. J. Neurophysiol. 103, 1410-1417.

de Graaf, T. A., de Jong, M. C., Goebel, R., van Ee, R., and Sack, A. T. (2011). On the functional relevance of frontal cortex for passive and voluntarily controlled bistable vision. Cereb. Cortex 10, 2322-2331.

Engel, E. (1956). The role of content in binocular resolution. Am. J. Psychol. 69, 87-91.

Epstein, R., and Kanwisher, N. (1998). A cortical representation of the local visual environment. Nature 392, 598601.

Fincher-Kiefer, R. (2001). Perceptual components of situation models. Mem. Cogn. 29, 336-343.

Forster, K. I., and Forster, J. C. (2003). DMDX: a windows display program with millisecond accuracy. Behav. Res. Methods Instrum. Comput. 35, 116-124.

Fox, R., and Check, R. (1968). Detection of motion during binocular rivalry suppression. J. Exp. Psychol. 78, 388395.

Fox, R., and Rasche, F. (1969). Binocular rivalry and reciprocal inhibition. Percept. Psychophys. 5, 215-217.

Gallese, V., and Lakoff, G. (2005). The brain's concepts: the role of the sensory-motor system in conceptual knowledge. Cogn. Neuropsychol. 22, 455-479.

Glenberg, A. M. (1997). What memory is for. Behav. Brain Sci. 20, 1-55.

Glenberg, A. M., and Kaschak, M. P. (2002). Grounding language in action. Psychon. Bull. Rev. 9, 558-565.

Hancock, S., Whitney, D., and Andrews, T. J. (2008). The initial interactions underlying binocular rivalry require visual awareness. J. Vis. 8, 1-9.

Harrad, R. A., McKee, S. P., Blake, R., and Yang, Y. (1994). Binocular rivalry disrupts stereopsis. Perception 23, 15-28.

Haynes, J. D., Deichmann, R., and Rees, G. (2005). Eye-specific effects of binocular rivalry in the human lateral geniculate nucleus. Nature 438, 496-499.

Holmes, D. J., Hancock, S., and Andrews, T. J. (2006). Independent binocular integration for form and colour. Vis. Res. 46, 665-677.

Hubel, D. H., and Wiesel, T. N. (1959). Receptive fields of single neurones in the cat's striate cortex. J. Physiol. 148, 574-591.

Isenberg, N., Silbersweig, D., Engelien, A., Emmerich, K., Malavade, K., Beati, B., Leon, A. C., and Stern, E. (1999). Linguistic threat activates the human amygdala. Proc. Natl. Acad. Sci. U.S.A. 96, 10456-10459.

Julesz, B., and Miller, J. E. (1975). Independent spatial frequency tuned channels in binocular fusion and rivalry. Perception 4, 125-143.

Kanwisher, N., McDermott, J., and Chun, M. (1997). The fusiform face area: a module in human extrastriate cortex specialized for face perception. J. Neurosci. 17, 4302-4311.

Kanwisher, N., Stanley, D., and Harris, A. (1999). The fusiform face area is selective for faces not animals. Neuroreport 10, 183-187.

Keliris, G. A., Logothetis, N. K., and Tolias, A. S. (2010). The role of the primary visual cortex in perceptual suppression of salient visual stimuli. J. Neurosci. 30, 12353-12365.

Klink, P. C., van Ee, R., Nijs, M. M., Brouwer, G. J., Noest, A. J., and van Wezel, R. J. (2008). Early interactions between neuronal adaptation and voluntary control determine perceptual choices in bistable vision. J. Vis. 8, 1-18.

Kovacs, I., Papathomas, T. V., Yang, M., and Feher, A. (1996). When the brain changes its mind: interocular grouping during binocular rivalry. Proc. Natl. Acad. Sci. U.S.A. 93, 15508-15511.

Lankheet, M. J. (2006). Unraveling adaptation and mutual inhibition in perceptual rivalry. J. Vis. 6, 304-310.

Lee, S. H., and Blake, R. (1999). Rival ideas about binocular rivalry. Vis. Res. 39, 1447-1454.

Lehmkuhle, S., and Fox, R. (1975). Effect of binocular rivalry suppression on the motion aftereffect. Vis. Res. 15, 855-859.

Leopold, D. A., and Logothetis, N. K. (1996). Activity changes in early visual cortex reflect monkeys' percepts during binocular rivalry. Nature 379, 549-553.
Leopold, D. A., and Logothetis, N K. (1999). Multistable phenomena: changing views in perception. Trends Cogn. Sci. 3, 254-264.

Levelt, W. (1965). On Binocular Rivalry. Assen: Royal VanGorcum.

Lin, Z., and He, S. (2009). Seeing the invisible: the scope and limits of unconscious processing in binocular rivalry. Prog. Neurobiol. 87, 195-211.

Logothetis, N. K. (1998). Single units and conscious vision. Philos. Trans. R. Soc. Lond. B Biol. Sci. 353, 1801-1818.

Logothetis, N. K., Leopold, D. A. and Sheinberg, D. L. (1996). What is rivalling during binocular rivalry? Nature 380, 621-624.

Logothetis, N. K., and Schall, J. D. (1989). Neuronal correlates of subjective visual perception. Science 245, 761-763.

McCarthy, G., Puce, A., Gore, J., and Allison, T. (1997). Face-specific processing in the human fusiform gyrus. J. Cogn. Neurosci. 9, 605-610.

Meteyard, L., Bahrami, B., and Vigliocco, G. (2007). Motion detection and motion verbs. Language affects low-level visual perception. Psychol. Sci. 18, 1007-1013.

Mitchell, J. F., Stoner, G. R., and Reynolds, J. H. (2004). Objectbased attention determines dominance in binocular rivalry. Nature 429, 410-413.

Ogle, K. N., and Wakefield, J. M. (1967) Stereoscopic depth and binocular rivalry. Vis. Res. 7, 89-98.

Ooi, T. L., and He, Z. J. (2003). A distributed intercortical processing of binocular rivalry: psychophysical evidence. Perception 32, 155-166.

O'Shea, R. P., Sims, A. J. H., and Govan, D. G. (1997). The effect of spatial frequency and field size on the spread of exclusive visibility in binocular rivalry. Vis. Res. 37, 175-183.

Papathomas, T. V., Kovacs, I., Feher, A., and Julesz, B. (1999). "Visual dilemmas: competition between eyes and between percepts in binocular rivalry," in Rutgers University Introduction to Cognitive Science, ed. E. Lepore (Basic Blackwell publishers), 263-294.

Pelekanos, V., and Moutoussis, K. (in press). The effect of language on visual contrast sensitivity. Perception.

Polonsky, A., Blake, R., Braun, J., and Heeger, D. (2000). Neuronal activity in human primary visual cortex correlates with perception during binocular rivalry. Nat. Neurosci. 3, 1153-1159.

Puce, A., Allison, T., Asgari, M., Gore, J. C., and McCarthy, G.
(1996). Differential sensitivity of human visual cortex to faces, letter strings, and textures: a functional magnetic resonance imaging study. J. Neurosci. 16, 5205-5215.

Pulvermüller, F. (1999). Words in the brain's language. Behav. Brain Sci. 22, 253-336.

Pulvermüller, F. (2005). Brain mechanisms linking language and action. Nat. Rev. Neurosci. 6, 576-582.

Rommetveit, R., Toch, H., and Svendsen, D. (1968). Effects of contingency and contrast contexts on the cognition of words. Scand. J. Psychol. 9, 138-144.

Sheinberg, D. L., and Logothetis, N. K. (1997). The role of temporal corti$\mathrm{cal}$ areas in perceptual organization. Proc. Natl. Acad. Sci. U.S.A. 94, 3408-3413.

Sobel, K. V., and Blake, R. (2002). How context influences predominance during binocular rivalry. Perception 31, 813-824.

Speer, N. K., Reynolds, J. R., Swallow, K. M., and Zacks, J. M. (2009). Reading stories activates neural representations of visual and motor experiences. Psychol. Sci. 20, 989-999.

Stanfield, R. A., and Zwaan, R. A. (2001). The effect of implied orientation derived from verbal context on picture recognition. Psychol. Sci. 12, 153-156.

Stocker, A. A., and Simoncelli, E. P. (2009). Visual motion aftereffects arise from a cascade of two isomorphic adaptation mechanisms. J. Vis. 9, 1-14.

Sutherland, N. S. (1961). Figural aftereffects and apparent size. Q. J. Exp. Psychol. 13, 222-228.

Tong, F. (2001). Competing theories of binocular rivalry: a possible resolution. Brain Mind 2, 55-83.

Tong, F., and Engel, S. A. (2001). Interocular rivalry revealed in the human cortical blind-spot representation. Nature 411, 195-199.

Tong, F., Meng, M., and Blake, R. (2006). Neural bases of binocular rivalry. Trends Cogn. Sci. 10, 502-511.

Tong, F., Nakayama, K., Vaughan, J. T., and Kanwisher, N. (1998). Binocular rivalry and visual awareness in human extrastriate cortex. Neuron 21, 753-759.

van Boxtel, J. J. A., Alais, D., and van Ee, R. (2008). Retinotopic and non-retinotopic stimulus encoding in binocular rivalry and the involvement of feedback. J. Vis. 8, 1-10.

van Ee, R., van Dam, L. C., and Brouwer, G. J. (2005). Voluntary control and the dynamics of perceptual bi-stability. Vis. Res. 45, 41-55. 
Walker, P. (1978). Binocular rivalry: central or peripheral selective processes? Psychol. Bull. 85, 376-389.

Wheatstone, C. (1838). Contributions to the physiology of vision. 1 . On some remarkable and hitherto unobserved phenomena of binocular vision. Philos. Trans. R. Soc. Lond. B Biol. Sci. 8, 371-394.

Wilson, H. R., Blake, R., and Lee, S. H. (2001). Dynamics of travelling waves in visual perception. Nature 412, 907-910.

Wohlgemuth, A. (1911). On the aftereffect of seen movement. Br. J. Psychol. Monogr. Suppl. 1, 1-117.
Wolfe, J. M. (1984). Reversing ocular dominance and suppression in a single flash. Vis. Res. 24, 471-478.

Yaxley, R. H., and Zwaan, R. A. (2007). Simulating visibility during language comprehension. Cognition 105, 229-236.

Zwaan, R. A. (2004). The immersed experiencer: toward an embodied theory of language comprehension. Psychol. Learn. Motiv. 44, 35-62.

Zwaan, R. A., Madden, C. J., Yaxley, R. H., and Aveyard, M. E. (2004). Moving words: dynamic representations in language comprehension. Cogn. Sci. 28, 611-619.
Zwaan, R. A., Stanfield, R. A., and Yaxley, R. H. (2002). Language comprehenders mentally represent the shapes of objects. Psychol. Sci. 13, 168-171.

Conflict of Interest Statement: The authors declare that the research was conducted in the absence of any commercial or financial relationships that could be construed as a potential conflict of interest.

Received: 01 August 2011; accepted: 22 December 2011; published online: 11 January 2012.
Citation: Pelekanos V, Rouman $D$ and Moutoussis K (2011) The effects of categorical and linguistic adaptation on binocular rivalry initial dominance. Front. Hum. Neurosci. 5:187. doi: 10.3389/fnhum.2011.00187

Copyright (c) 2012 Pelekanos, Roumani and Moutoussis. This is an openaccess article distributed under the terms of the Creative Commons Attribution Non Commercial License, which permits non-commercial use, distribution, and reproduction in other forums, provided the original authors and source are credited. 